MATEC Web of Conferences 31, 16001 (2015)

DOI: $10.1051 /$ matecconf/ 20153116001

(C) Owned by the authors, published by EDP Sciences, 2015

\title{
Research on Method of Character Recognition Based on Hough Transform and RBF Neural Network
}

\author{
Zhang Yin ${ }^{1,2}$, Chen Guangyu ${ }^{1}$, Zhang Lei ${ }^{1}$, Li Huixin ${ }^{1}$, Wang Dongyun ${ }^{1}$ \\ 1 Qinhuangdao Institute of Technology in Qinhuangdao, Hebei, China \\ ${ }^{2}$ College of Mechanical Engineering Yanshan University in Qinhuangdao, Hebei, China
}

\begin{abstract}
A method of character recognition based on Hough transform and RBF neural network is proposed through research on weight accumulation algorithm of Hough transform. According to the feature of characters' structure by using the duality of point-line Hough transform was done. In this method, the number of the points on the same line in parameter space and the position coordinates of the elements in image mapping space were taken to RBF neural network recognition system as characteristic input vector. It reduced the dimension of character feature vector and reflected the overall distribution of character lattice and the essential feature of character shape. The simulation results indicated there were some merits in this improved method: capability of recognition is strong, the quantity of calculation is small, and the speed of calculation is quick.
\end{abstract}

\section{Introduction}

Hough transform is one of image edge detection technologies. Any arbitrary analytic curve of image space can be identified and detected with Hough transform (1, 2). There are some merits for Hough transform: it is not sensitive to the local defect of image; it is robust to random noise and it is suitable for parallel processing and real-time applications. So it is favored by scholars researched on image processing, pattern recognition and computer vision $(3,4)$.

In this paper through analysis some problems of the existing standard Hough transform, a character feature extraction method based on improved Hough transform was presented. Weight accumulation is used to overcome the interference to which binary accumulation of peak detection bring in Hough transform. It is applied to the recognition system of characters and numbers that the improved Hough transform method combined with RBF neural network. Contrast to the previous method, some merits was presented: capability of recognition is strong, the quantity of calculation is small, and the speed of calculation is quick.

\section{Optimization of Hough transform lgorithm}

\subsection{Problem of standard Hough transform in application}

In standard Hough transform, generally binary accumulation method was used to accumulate evidence of parameter space. That means the element in accumulation unit is added one. Therefore after every unit was done in the whole parameter space, the accumulation matrix in which accumulation value was recorded was derived $(5,6)$. As the Figure1 (a) show, the points located on same line in image space intersect at one point in the parameter space after Hough transform and the transform curve presents the radiation distribution around the intersection. Because of this distribution characteristics around intersection, accumulation value of parameter space present butterfly shape distribution around accumulation peak value in arbitrary value accumulation mode, it means that accumulation value of accumulation matrix take the accumulate peak value as the center. The difference value between accumulation value at peak with accumulation value near peak is not large which is shown in Figure 1 (b). It is difficult to set threshold of parameter space, measure and extract peak value. It notes that the part accumulation figure of accumulation matrix was derived from $9 * 9$ accumulation unit which is near to accumulation peak value in whole accumulation matrix $(7,8)$

\subsection{Optimization of Hough transform}

From above figures, it can be seen that the mapping curves of parameter space were more intensive in common voting region, and relative scattered in other regions. The sample variance in each accumulation unit is taken as criterion; by using sample variance the weight of each accumulation unit was accumulated to eliminate interference of the accumulation unit near the accumulation peak.

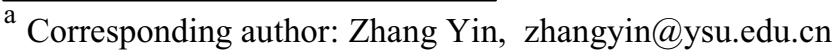




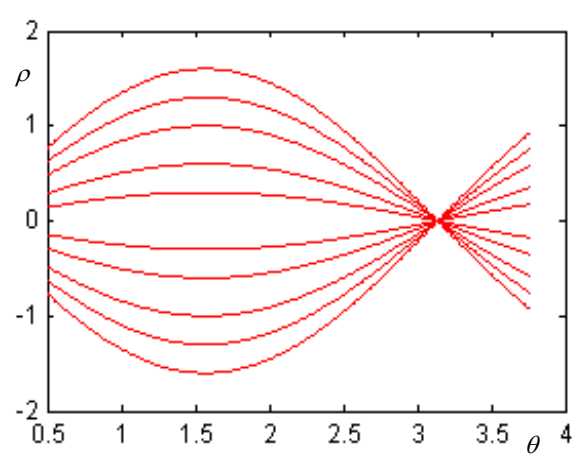

Fig1 (a) mapping figure in parameter space

$$
\left[\begin{array}{ccccccccc}
0 & 0 & 0 & 0 & 0 & 0 & 0 & 0 & 0 \\
0 & 0 & 0 & 0 & 0 & 0 & 0 & 0 & 0 \\
1 & 0 & 0 & 0 & 0 & 0 & 0 & 1 & 1 \\
2 & 3 & 1 & 0 & 0 & 0 & 1 & 2 & 2 \\
2 & 3 & 5 & 8 & 10 & 9 & 6 & 3 & 2 \\
3 & 3 & 4 & 2 & 0 & 1 & 2 & 2 & 3 \\
2 & 1 & 0 & 0 & 0 & 0 & 1 & 2 & 3 \\
0 & 0 & 0 & 0 & 0 & 0 & 0 & 0 & 1 \\
0 & 0 & 0 & 0 & 0 & 0 & 0 & 0 & 0
\end{array}\right]
$$

Fig1 (b) part accumulation matrix

The process of weights accumulation is as follow:

First parameter space was quantified. Then according to the results of quantification, accumulation matrix $\mathrm{A}$ is defined. The element $\rho_{\text {in }}$ of transformed matrix $\rho$ was accumulated in unit $A(m, n), \rho_{\text {in }}$ which satisfied all conditions is called sample of accumulation unit $A(m, n)$.

Second the sample standard deviation matrix $\mathrm{S}$ was defined according to accumulation matrix A. for example the accumulation unit $A(m, n)$ is:

$$
\rho_{n}^{m}=\left[\rho_{i_{1} n}, \rho_{i_{2} n}, \ldots, \rho_{i_{i} n}\right]^{T}
$$

$\rho_{n}^{m}$ is sample vector of accumulation unit $A(m, n)$, the sample standard deviation $S(m, n)$ is derived as follow:

Threshold $T_{1}$ was set; the number of element of $\rho_{n}^{m}$ is compared with set Threshold ${ }^{T_{1}}$, if larger than ${ }^{T_{1}}$,

$$
S(m, n)=\operatorname{std}\left(\rho_{n}^{m}\right)
$$

Otherwise

$$
S(m, n)=\mathrm{C}
$$

$\mathrm{C}$ is a constant, and it is enough larger than the sample standard deviation which is derived through equation (2). So the standard deviation matrix $S(m, n)$ can be derived with this method.

At last, according to the standard deviation matrix S, weight of accumulation unit is accumulated, the weight is

$$
w_{t}^{\prime}=\frac{S_{\min }}{S(m, n)}
$$

$S_{\min }$ is the minimum sample standard deviation of whole parameter space. $S(m, n)$ is sample standard deviation of accumulation unit.

Accumulation unit $A(m, n)$ is taken as example; the accumulation value is calculated with equation (5)

$$
A(m, n)=\sum_{i=1}^{l} 1 \cdot w_{i}=\sum_{i=1}^{l} 1 \cdot\left(\frac{S_{\min }}{S(m, n)}\right)^{2}
$$

The accumulation matrix is calculated after simulation. The part weight accumulation matrix is derived with $9 \times 9$ accumulated unit which were chosen around accumulation peak as figure 2 :

$$
\left[\begin{array}{ccccccccc}
0 & 0 & 0 & 0 & 0 & 0 & 0 & 0 & 0 \\
0 & 0 & 0 & 0 & 0 & 0 & 0 & 0 & 0 \\
0 & 0 & 0 & 0 & 0 & 0 & 0 & 0 & 0 \\
0 & 0 & 0 & 0 & 0 & 0 & 0 & 0 & 0 \\
0 & 0 & 0.13 & 1.05 & 10 & 1.34 & 0.24 & 0 & 0 \\
0 & 0 & 0.05 & 0 & 0 & 0 & 0 & 0 & 0 \\
0 & 0 & 0 & 0 & 0 & 0 & 0 & 0 & 0 \\
0 & 0 & 0 & 0 & 0 & 0 & 0 & 0 & 0 \\
0 & 0 & 0 & 0 & 0 & 0 & 0 & 0 & 0
\end{array}\right]
$$

Fig. 2 The part weight accumulation matrix

\section{The simulation of character recognition}

\subsection{The mathematical model}

First it is assumed that character or number which is to be recognized is not polluted. The image of character or number is coded with $16 \times 16$ lattice. The squares of

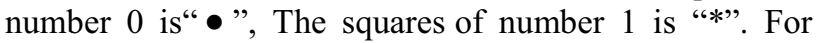
example, the character $\mathrm{Z}$ is shown in figure 3, and the matrix of $Z$ is shown in figure 4 


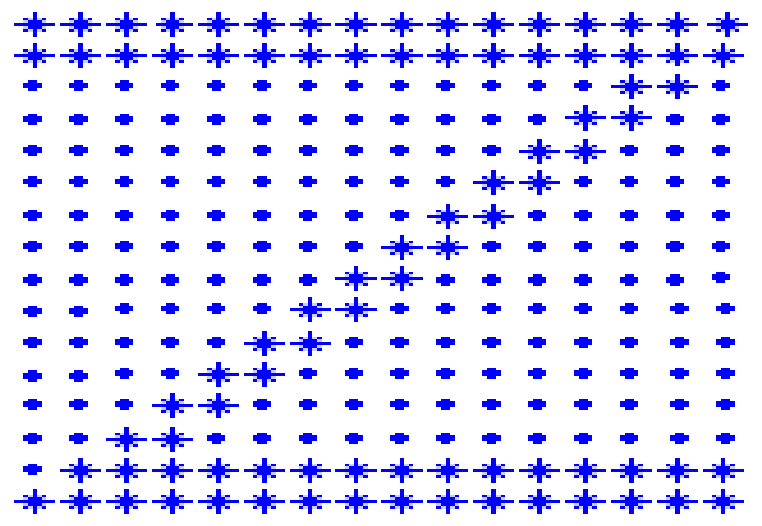

Fig.3 The coding of letter $Z$

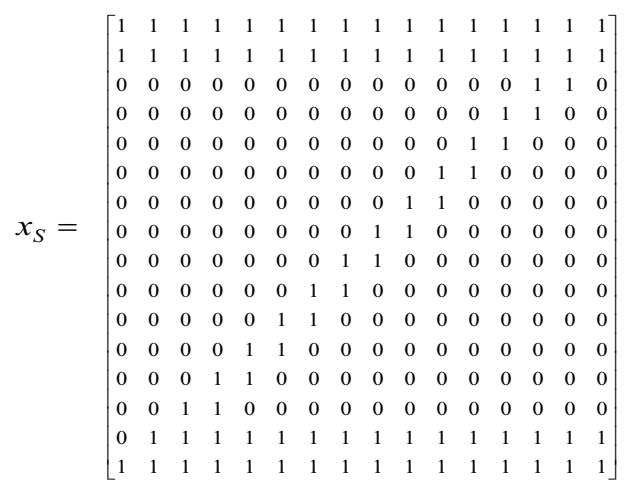

Fig.4 The matrix of letter $\mathrm{Z}$

As input, it is turned into $256 \times 1$ column vector, mapping expected output is $36 \times 1$ column vector, corresponding character position is 1 , others is 0 , corresponding output is

$$
\begin{aligned}
& Y_{S}=[0000000000000000000 \\
& 00000000001000000000000]^{T}
\end{aligned}
$$

Similarly the results of other 35 numbers or characters can be derived. Because Normal random noise is a random number between -1 to 1 , so $0 \sim 0.4$ noise was added to 36 group of samples to simulate real data which polluted with noise. The sample space composed of polluted data and expected data transferred to RBF Neural network recognition system to train.

\subsection{Simulation and analysis the experiment result}

According to the above method, 36 character sample of dot matrix are selected, then Hough transform is done and the average value $\mathrm{Q}$ of $A(\rho, \theta)=0$ in the sample cumulative array $A(\rho, \theta)$ of 36 characters is calculated. It is show that the statistic result of $\mathrm{Q}$ is about 160 . Therefore, in a $16 \times 16$ dot matrix of character, after the elements of $A(\rho, \theta)=0$ were removed, the feature number of accumulator array $A(\rho, \theta)$ is reduced from $16 \times 16=256$ to about 100 .

The position coordinates of the elements of the accumulation array $A(\rho, \theta)$ in the corresponding image mapping space were input to another array $B(x, y)$, the number of feature of $A(\rho, \theta)$ and $B(x, y)$ is up to 200. Similar method was taken; number of collinear points of $A(\rho, \theta)>2$ in accumulation array $A(\rho, \theta)$ was statistic. The results show that the average value of collinear points of $A(\rho, \theta)>2$ in accumulation array $A(\rho, \theta)$ is about 60. Finally, accumulate array is sorted in descending order, 60 coordinates element of array and its mapping position in the image mapping space, totally 120 elements as the feature vector, were input to RBF neural network recognition system in sequence.

Data test analysis were done in case with $0.1,0.2,0.30 .4$ random noise interference respectively as shown in Figure 5, where the vertical axis is the error square sum of output vector, the horizontal axis represents the number of test data. in Figure 5 it can be concluded that when the smaller interference was input to system such as $0.1,0.2$, the output can be completely follow the expected value, as shown in Figure 5(a), $5(b)$ is shown but when the bigger interference was input to system such as $0.3,0.4$ The output cannot completely follow the expected value, namely appeared the recognition error, as shown in Figure 5(c), 5(d) is shown.

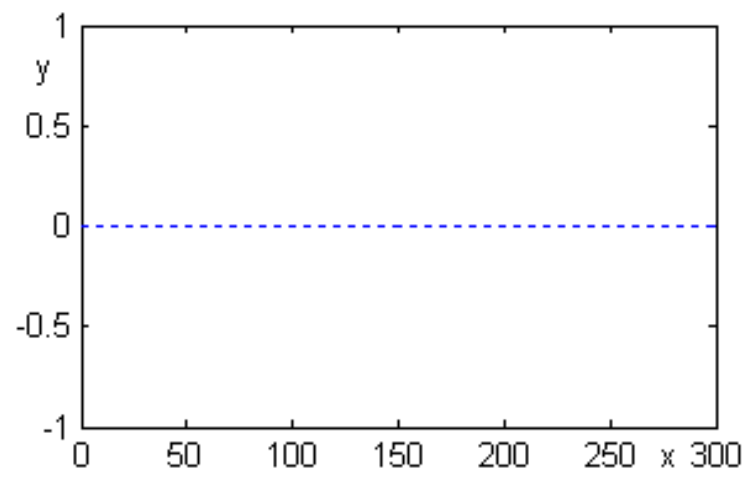

Fig.5 (a) The disturbance is 0.1

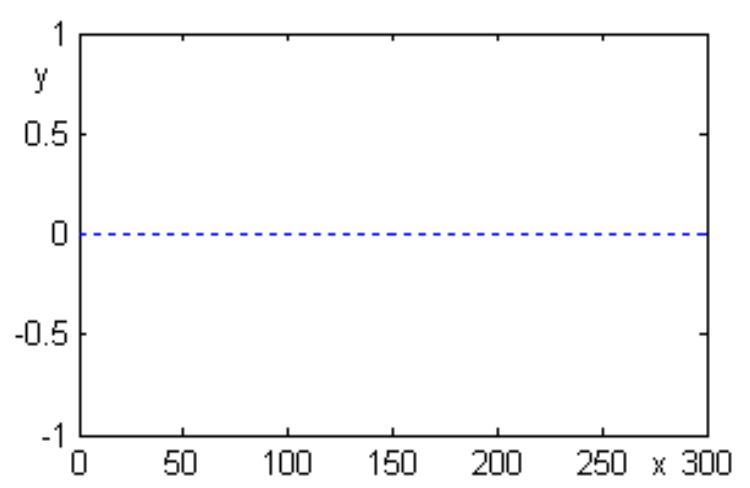

Fig.5 (b) The disturbance is 0.2 


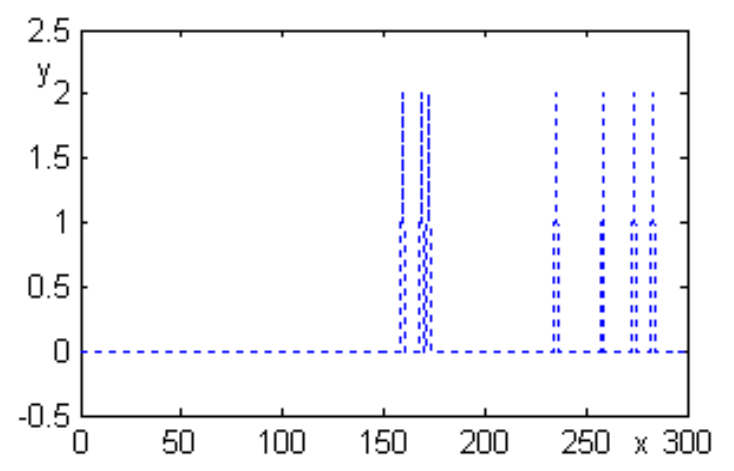

Fig.5(c) The disturbance is 0.3

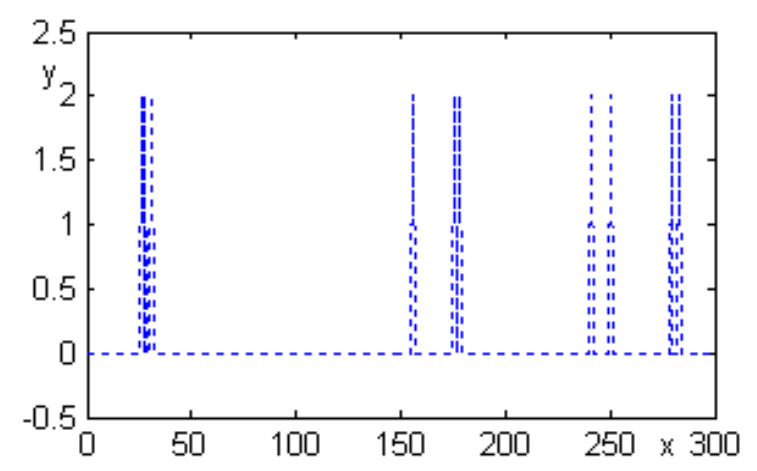

Fig.5 (d) The disturbance is 0.4

In order to understand the recognition ability of the improved algorithm, the recognition rate changes with the amount of interference was made, as shown in Figure 6 .

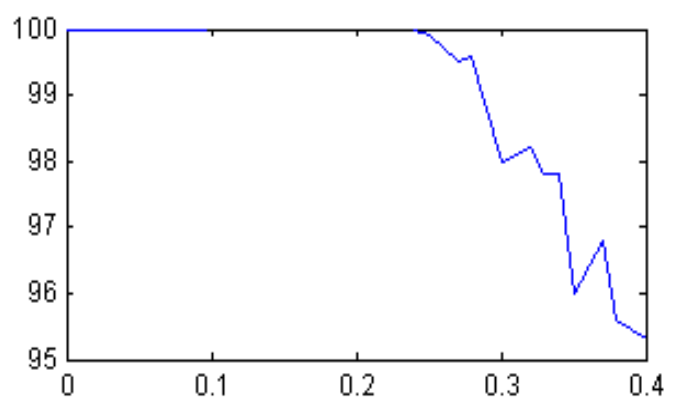

Fig. 6 The figure of arithmetic recognition ratio with the variety of disturbances

From the figure it can be seen, when the random noise added in the disturbance is less than 0.24 ; the system can completely identify the input data. Along with the increase of disturbance, the correct recognition rate began to decline, however, when the random noise disturbance reached 0.4 , the recognition rate can still reach more than $96 \%$. It indicated that the recognition ability of this improved method based on Hough transform and RBF neural network is strong, it has the good anti-interference ability; and can satisfy the purpose of system design

Simulation studies show that this method of the Hough transform which is applied to character recognition feature extraction has some merits, such as good recognition ability, small amount of calculation, fast extraction speed etc, its performance is superior to the original identification technology. And it provides technical guarantee for the wide application of character feature extraction and RBF neural network.

\section{Conclusion}

In this paper, a method of character feature extraction based on pattern recognition and Hough transform is presented, According to the characteristic of characters' structure by using the duality of point-line Hough transform was done. In this method, the number of the points on the same line in parameter space and the position coordinates of the elements in picture mapping space were taken to RBF neural network recognition system as characteristic input vector. It reduced the dimension of character feature vector, reflects the overall distribution of character lattice and the essential characteristic of character shape. The results show that this method is simple and easy, and has better stability. Tt is effective to realize the character feature extraction.

\subsection{Captions/numbering}

Captions should be typed in 9-point Times. They should be centred above the tables and flush left beneath the figures.

\section{Acknowledgment}

This project is supported by National Natural Science Foundation of China (Grant No. 51375422) and The Education Department of Hebei Province (Grant No. QN20132017).

\section{References}

1. ZHANG Zhifeng;XU Benxiang;JIN Wenbiao. Hough straight line detection algorithm based on two- colored pixe. Journal of Computer Applications. 2014, 34:196-198

2. Zhang Jia;Chen Jie;Dou Lihua. Shape detection based on hough transform in small optical tracking system.Experimental technology and magagement. 2014. 4 vol.31: 89-92.

3. ZHANG Guo-ying;CHENG Yi-yu;ZHU Hong. Detection of Linear target based on improved Hough transform. Computer engineering and design. 536540.

4. Song Xiaoyu;Yuan Shuai;Guo Hanbing;Liu Jifei. Pattern identification algorithm with adaptive threshold interval based extended Hough transform. Chinese Journal of Scientific Instrument. 2014 NO.5 Vol.351109-1117

5. ADAM H. Real-time detection of lines and grids[M]. Springer Press, 2013

6. Liu Hong-min, Wang Zhi-heng, Deng Chao. Polygon detection based on meta-representation[J]. Acta Automatica Sinaca, 2011,37(9):1020-1058

7. YANG D L, LU CH H, ZHANG J L, et al. Edge detection in SAR images based on ROEWA and 
Hough transfor[J]. Journal of Electronic Measurement andInstrument, 2013, 27 ( 6) , 543-548

8. ZHOU F, YANG CH, WANG $\mathrm{CH} \mathrm{G}$, et al. Circle detection and its number identification in complex condition based on random Hough transform $[\mathrm{J}]$. Chinese Journal of Scientific Instrument. 2013, 34( 3): 622-628 